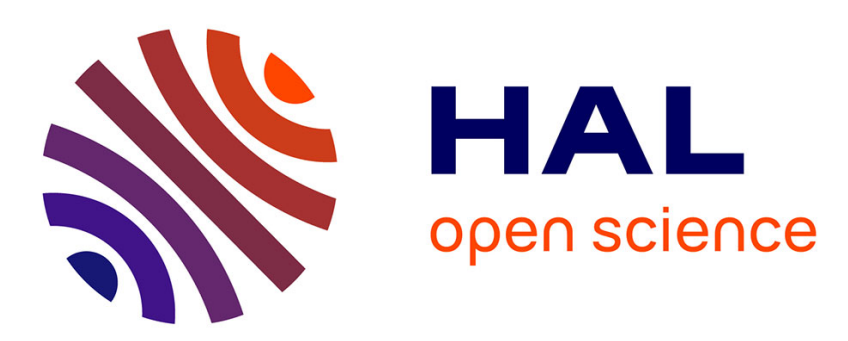

\title{
MagnetoHemoDynamics in Aorta and Electrocardiograms
}

Vincent Martin, Agnès Drochon, Odette Fokapu, Jean-Frédéric Gerbeau

\section{To cite this version:}

Vincent Martin, Agnès Drochon, Odette Fokapu, Jean-Frédéric Gerbeau. MagnetoHemoDynamics in Aorta and Electrocardiograms. Physics in Medicine and Biology, 2012, 57 (10), pp.3177-3195. 10.1088/0031-9155/57/10/3177 . inria-00628032v2

\section{HAL Id: inria-00628032 https://hal.inria.fr/inria-00628032v2}

Submitted on 23 May 2012

HAL is a multi-disciplinary open access archive for the deposit and dissemination of scientific research documents, whether they are published or not. The documents may come from teaching and research institutions in France or abroad, or from public or private research centers.
L'archive ouverte pluridisciplinaire HAL, est destinée au dépôt et à la diffusion de documents scientifiques de niveau recherche, publiés ou non, émanant des établissements d'enseignement et de recherche français ou étrangers, des laboratoires publics ou privés. 


\title{
MagnetoHemoDynamics in the Aorta and Electrocardiograms
}

\author{
Vincent Martin ${ }^{1,4}$ Agnès Drochon ${ }^{2}$, Odette Fokapu ${ }^{2,3}$ and \\ Jean-Frédéric Gerbeau ${ }^{4}$ \\ ${ }^{1}$ University of Technology of Compiègne (UTC), LMAC, GI, Royallieu, BP 20529, 60205 \\ Compiègne, France. \\ ${ }^{2}$ UTC, UMR 7338, GB, Royallieu, BP 20529, 60205 Compiègne, France. \\ ${ }^{3}$ Université de Picardie Jules Vernes, 80025 Amiens, France. \\ ${ }^{4}$ INRIA Paris-Rocquencourt, BP 105, 78153 Le Chesnay, France. \\ E-mail: Vincent.Martin@utc.fr, Agnes.Drochon@utc.fr, Odette.Fokapu@utc.fr, \\ Jean-Frederic.Gerbeau@inria.fr.
}

\begin{abstract}
This paper addresses a complex multi-physical phenomemon involving cardiac electrophysiology and hemodynamics. The purpose is to model and simulate a phenomenon that has been observed in MRI machines: in the presence of a strong magnetic field, the $T$-wave of the electrocardiogram (ECG) gets bigger, which may perturb ECG-gated imaging. This is due a magnetohydrodynamic (MHD) effect occurring in the aorta. We reproduce this experimental observation through computer simulations on a realistic anatomy, and with a three-compartment model: inductionless magnetohydrodynamic equations in the aorta, bidomain equations in the heart and electrical diffusion in the rest of the body. These compartments are strongly coupled and solved using finite elements. Several benchmark tests are proposed to assess the numerical solutions and the validity of some modeling assumptions. Then, ECGs are simulated for a wide range of magnetic field intensities (from 0 to 20 Tesla).
\end{abstract}

Keywords: Blood flow, electrophysiology, Magnetohydrodynamics (MHD), MRI, finite elements.

\section{Introduction}

The interaction between the blood flow and a strong magnetic field generates an induced electric field in the body. In a Magnetic Resonance Imaging (MRI) machine, this "MagnetoHemoDynamic" (MHD) effect mainly occurs in the aortic arch, because the blood velocity there is large and roughly orthogonal to the magnetic field in this location. The induced voltage may alter the electric potential measured in the ElectroCardioGrams (ECG). It has indeed been observed that it makes the $T$-wave larger [9], which in turn may perturb the ECG-gated image acquisition in MRI. This effect gets worse with higher magnetic field, which is the current trend of MRI (from typically 3 Tesla nowadays for humans, it may reach 10 Tesla in the future). Different ways of overcoming this gating problem have been proposed, for instance using VectoCardioGrams (VCG) [5], or acoustic cardiac signal [8], ECG signal processing [31]. However, a better understanding and prediction of the MHD effect would have many potential benefits. First, it may help improve the quality of the ECG, which is not only used for gating but also for clinical purposes, in particular for interventional MRI or MRI stress tests. Second, the MHD artifact might be used to infer some physical properties of the system, using inverse problem techniques. This paper is devoted to the modeling and numerical simulation of this phenomenon, and can be viewed as a first step in this direction. 
The interaction of the aortic blood flow and a magnetic field has already been investigated in the literature. An overview of the in vitro experiments, in vivo studies as well as MHD calculations in blood flows can be found in [1]. Gaffey et al [9] and Tenforde et al [30] observed animal ECG alterations, manifested mainly as $T$-wave elevations. Later, the same group provided some simulations of the voltage and current distributions, and the resulting reduction in blood flow, by means of a two-dimensional finite element analysis in two sections of the ascending and descending aorta with a conductive wall [16]. They studied the case of a steady state flow with an external magnetic field transverse to the ascending and descending aorta. More recently, Sahakian et al published a series of papers where the magnetohydrodynamic effect during cardiac MRI was investigated both experimentally [23] and by computer simulations, using the Comsol Multiphysics software [25, 24]. Computer simulations of the MHD effect, with experimental validations, are also proposed in [15]. Another group addressed the problem of ECG simulations in the presence of the magnetic field [13] using the closed-form solution of Gold [12], which corresponds to a steady, fully developed flow of a conducting fluid in a straight cylinder subjected to a transverse magnetic field. In this approach, the aortic arch was divided into slices. In each slice, Gold's solution was used, with a different angle for the magnetic field. The MHD signal was computed from these slices via a transfer function and then directly added to the ECG waveform that was taken from the ECGSIM software [14].

In the present study, state-of-art mathematical models are considered in each physical compartment of the problem on a realistic anatomy: bidomain equations for the cardiac electrophysiology; inductionless MHD equations for the aortic blood flow; electrostatic equations in the torso, which includes bones and lungs. In contrast to what is usually done in the literature, these three compartments are strongly coupled. This allows us to evaluate the possible interaction between the electric potential induced in the aorta and the extracellular potential in the myocardium. The present article significantly enriches the preliminary results presented in [19]. It contains many details about the numerical methods and several new results. Due to the complexity of the model and the multiphysical coupling, a particular effort has been placed on verifying the computer simulations: several new test cases are proposed to assess the MHD solutions and the coupling algorithm with the torso. In addition, a numerical justification of the inductionless approximation of the MHD equations in the blood flow regime is presented. Finally, new numerical simulations of ECG are performed for a wide range of magnetic fields (up to 20 Tesla) and new conclusions are drawn about the link between the flow rate, the magnetic field and the ECG perturbation.

\section{Models}

Let $\Omega$ be a domain representing the whole body (Fig. 1) partitioned into three subdomains, the aorta $\Omega_{\mathrm{A}}$, the heart $\Omega_{\mathrm{H}}$ and the complement $\Omega_{\mathrm{T}}$, hereafter sometimes called "torso" for the sake of brevity: $\bar{\Omega}=\overline{\Omega_{\mathrm{A}}} \cup \overline{\Omega_{\mathrm{H}}} \cup \overline{\Omega_{\mathrm{T}}}$, where $\bar{\omega}$ denotes the closure of an open domain $\omega$, and $\partial \omega$ its boundary. The boundary of the aorta is assumed to be entirely included in the torso and divided into an inlet, four outlets and a wall: $\partial \Omega_{\mathrm{A}}=\Gamma_{\text {inlet }} \cup \Gamma_{\text {wall }} \cup \Gamma_{\text {outlets }} \subset \bar{\Omega}_{\mathrm{T}}$. The inlet is a planar cut in the ascending aorta. The first three outlets $\Gamma_{\text {outlet }_{i}}, i=1,2,3$, are respectively a section of the brachiocephalic, the left common carotid, and the left subclavian arteries. The outlet $\Gamma_{\text {outlet }_{4}}$ is a section of the abdominal aorta (Fig. 7). The heart boundary is included in the torso $\partial \Omega_{\mathrm{H}} \subset \bar{\Omega}_{\mathrm{T}}$, thus the boundary of the torso is partitioned as follows: $\partial \Omega_{\mathrm{T}}=\partial \Omega_{\mathrm{A}} \cup \partial \Omega_{\mathrm{H}} \cup \Gamma_{\text {ext }}$, where $\Gamma_{\text {ext }}$ is the body surface. Throughout the article, $\boldsymbol{n}$ will denote a normal unit vector on given surfaces.

Let us briefly review the main stages of the electrical activity of the myocardium. During the 
depolarization, an electrical activation wavefront propagates throughout the heart. This generates an electric potential differences in $\Omega_{\mathrm{T}}$. The recording of these potential differences gives the ECG. The first event seen in the ECG, called the $P$-wave, corresponds to the depolarization of the atria. It is not modeled in the present study. Next, the ventricles are depolarized, which corresponds to the $Q R S$-complex in the ECG. The $R$-wave, which is the largest positive deflection in the lead $I$, is used for MRI gating. This depolarization triggers the mechanical contraction of the myocardium, which ejects the blood from the left ventricle into the aorta (systole). Next, the ventricles return to their rest state (repolarization), which gives the last deflection of the ECG, called the $T$-wave. The peak flow in the aortic arch, and therefore the peak MHD effect, takes place after the $S$-wave (Fig. 1).

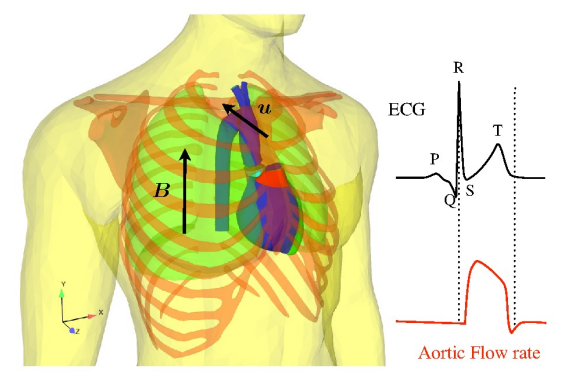

Figure 1. Left: the three subdomains (aorta, heart and rest of the body, including some bones and the lungs), schema of the magnetic field $\boldsymbol{B}$ and velocity field $\boldsymbol{u}$ in the aortic arch. Right: sketch of the lead $I$ of the ECG and aortic flow rate.

\subsection{MHD in the aorta}

In the presence of an electric field $\boldsymbol{E}$ and a magnetic field $\widetilde{\boldsymbol{B}}$, Ohm's law in the blood is written $\boldsymbol{j}=\boldsymbol{\sigma}_{\mathrm{bl}}(\boldsymbol{E}+\boldsymbol{u} \times \widetilde{\boldsymbol{B}})$, where $\boldsymbol{j}$ denotes the current density, $\boldsymbol{\sigma}_{\mathrm{bl}}$ the electrical conductivity of the blood and $\boldsymbol{u}$ the blood velocity. Ignoring the displacement current $\epsilon_{0} \partial_{t} \boldsymbol{E}$, the Maxwell-Ampère equation gives another expression of the current density:

$$
j=\operatorname{curl} \frac{\widetilde{B}}{\mu},
$$

where $\mu$ is the magnetic permeability, supposed to be that of the vacuum $\left(\mu=\mu_{0}=4 \pi 10^{-7} \mathrm{H} \mathrm{m}^{-1}\right)$. Inserting these two expressions in the Maxwell-Faraday equation

$$
\partial_{t} \widetilde{\boldsymbol{B}}+\operatorname{curl} \boldsymbol{E}=0,
$$

and coupling them to the incompressible Navier-Stokes equations that describe blood flow in large arteries [7], we obtain the usual MHD equations:

$$
\left\{\begin{aligned}
\rho\left(\frac{\partial \boldsymbol{u}}{\partial t}+\boldsymbol{u} \cdot \nabla \boldsymbol{u}\right)-\eta \Delta \boldsymbol{u}+\nabla p & =\frac{1}{\mu_{0}} \operatorname{curl} \widetilde{\boldsymbol{B}} \times \widetilde{\boldsymbol{B}}, \\
\operatorname{div} \boldsymbol{u} & =0, \\
\frac{\partial \widetilde{\boldsymbol{B}}}{\partial t}+\operatorname{curl}\left(\frac{1}{\mu_{0} \boldsymbol{\sigma}_{\mathrm{bl}}} \operatorname{curl} \widetilde{\boldsymbol{B}}\right) & =\operatorname{curl}(\boldsymbol{u} \times \widetilde{\boldsymbol{B}}),
\end{aligned}\right.
$$


where $p$ denotes the pressure of the blood, $\eta$ its viscosity and $\rho$ its density. The right-hand side of (3) ${ }_{1}$ corresponds to the Lorentz force $\boldsymbol{j} \times \widetilde{\boldsymbol{B}}$, with $\boldsymbol{j}$ given by (1).

System (3) is typically used for fluids with high electrical conductivity, like liquid metals, but is not very suitable for blood flows. Indeed, the magnetic Reynolds number $R m$, which is the dimensionless ratio of magnetic advection to magnetic diffusion, is extremely small in that case (typically of order $10^{-8}$ during systole, cf. Table A2). Thus, the magnetic field is almost unaffected by the blood flow. In this regime, it is enough to assume that $\widetilde{\boldsymbol{B}}$ is equal to $\boldsymbol{B}$, the external imposed magnetic field, neglecting the MHD effect on $\boldsymbol{B}$. In an MRI device, $\boldsymbol{B}$ is mainly homogeneous and constant in time. Thus, if $\widetilde{\boldsymbol{B}} \approx \boldsymbol{B}$, the Maxwell-Faraday equation (2) gives curl $\boldsymbol{E}=0$, and there exists an electric potential $\phi_{\mathrm{A}}$ such that $\boldsymbol{E}=-\boldsymbol{\nabla} \phi_{\mathrm{A}}$. With this expression of $\boldsymbol{E}$, and neglecting again the variations of $\widetilde{\boldsymbol{B}}$ caused by the induction, Ohm's law becomes $\boldsymbol{j}=\boldsymbol{\sigma}_{\mathrm{bl}}\left(-\boldsymbol{\nabla} \phi_{\mathrm{A}}+\boldsymbol{u} \times \boldsymbol{B}\right)$. Using this relation in the Lorentz force, and writing the conservation of the electrical charges $\operatorname{div} \boldsymbol{j}=0$, we obtain a simplified form of MHD equations in $\Omega_{\mathrm{A}}$, sometimes called inductionless MHD equations,

$$
\left\{\begin{aligned}
\rho\left(\frac{\partial \boldsymbol{u}}{\partial t}+\boldsymbol{u} \cdot \boldsymbol{\nabla} \boldsymbol{u}\right)-\eta \Delta \boldsymbol{u}+\boldsymbol{\nabla} p & =-\boldsymbol{\sigma}_{\mathrm{bl}} \boldsymbol{\nabla} \phi_{\mathrm{A}} \times \boldsymbol{B}+\boldsymbol{\sigma}_{\mathrm{bl}}(\boldsymbol{u} \times \boldsymbol{B}) \times \boldsymbol{B}, \\
\operatorname{div} \boldsymbol{u} & =0, \\
\operatorname{div}\left(\boldsymbol{\sigma}_{\mathrm{bl}} \boldsymbol{\nabla} \phi_{\mathrm{A}}\right) & =\operatorname{div}\left(\boldsymbol{\sigma}_{\mathrm{bl}} \boldsymbol{u} \times \boldsymbol{B}\right) .
\end{aligned}\right.
$$

In the blood flow regime, system (4) is an excellent approximation of (3) for a wide range of $\boldsymbol{B}$, as will be shown by numerical simulations in Section 4.1. Moreover, it is numerically better conditioned, less expensive to solve, and it provides the electric potential directly, which is the quantity of interest for the ECG.

Fluid boundary conditions. A time dependent plug flow is applied on $\Gamma_{\text {inlet }}$, a no-slip condition is applied on $\Gamma_{\text {wall }}$, and a pressure is applied on $\Gamma_{\text {outlet }_{\mathrm{i}}, i=1, . ., 4}$. The outlet pressures $\bar{p}_{i}$ are obtained by coupling three-element Windkessel models to the Navier-Stokes equations: $\bar{p}_{i}=\pi_{i}+R_{p, i} Q_{i}$ where

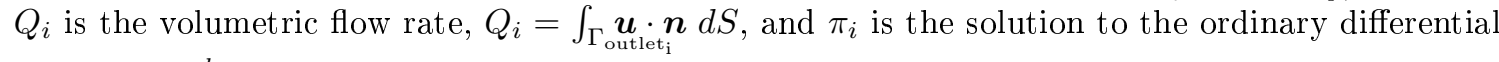
equation $C_{i} \frac{d \pi_{i}}{d t}+\frac{\pi_{i}}{R_{d, i}}=Q_{i}$. The parameters $R_{d, i}, R_{p, i}$ and $C_{i}$ represent the distal and proximal resistances and the capacitance $C_{i}$ respectively (values taken from [21]).

\subsection{Electrophysiology in the heart}

We denote by $\phi_{\mathrm{H}}$ and $V_{\mathrm{m}}$ the extracellular potential and the transmembrane potential, and by $\boldsymbol{\sigma}_{\mathrm{i}}$ and $\sigma_{\mathrm{e}}$ the intra- and extracellular conductivity tensors. In the heart $\Omega_{\mathrm{H}}$, the electrical activity is governed by the bidomain equations [27, 28]:

$$
\left\{\begin{aligned}
A_{\mathrm{m}}\left(C_{\mathrm{m}} \frac{\partial V_{\mathrm{m}}}{\partial t}+I_{\mathrm{ion}}\left(V_{\mathrm{m}}, w\right)\right)-\operatorname{div}\left(\boldsymbol{\sigma}_{\mathrm{i}} \boldsymbol{\nabla} V_{\mathrm{m}}\right)-\operatorname{div}\left(\boldsymbol{\sigma}_{\mathrm{i}} \boldsymbol{\nabla} \phi_{\mathrm{H}}\right) & =A_{\mathrm{m}} I_{\mathrm{app}}, \\
-\operatorname{div}\left(\left(\boldsymbol{\sigma}_{\mathrm{i}}+\boldsymbol{\sigma}_{\mathrm{e}}\right) \boldsymbol{\nabla} \phi_{\mathrm{H}}\right)-\operatorname{div}\left(\boldsymbol{\sigma}_{\mathrm{i}} \boldsymbol{\nabla} V_{\mathrm{m}}\right) & =0,
\end{aligned}\right.
$$

where $A_{\mathrm{m}}$ is a constant representing the rate of membrane area per volume unit and $C_{\mathrm{m}}$ the membrane capacitance per area unit. The term $I_{\text {ion }}\left(V_{\mathrm{m}}, w\right)$ represents the ionic current through the cell membranes, and $I_{\text {app }}$ is a given source term. The dynamics of the ionic variable $w$ and the ionic current $I_{\text {ion }}$ are governed by the phenomenological two-variable model proposed by Mitchell and Schaeffer in [20]. The ventricles are subdivided into four different regions where the coefficient governing the Action Potential Duration takes different values. The atria are not considered here, 
which explains the absence of the $P$-wave in our ECGs. The initial activation starts from the septum. Further details can be found in [3]. On the boundary of the heart, the intracellular medium is isolated:

$$
\boldsymbol{\sigma}_{\mathrm{i}} \boldsymbol{\nabla} V_{\mathrm{m}} \cdot \boldsymbol{n}+\boldsymbol{\sigma}_{\mathrm{i}} \boldsymbol{\nabla} \phi_{\mathrm{H}} \cdot \boldsymbol{n}=0 \quad \text { on } \quad \partial \Omega_{\mathrm{H}} .
$$

\subsection{Electric diffusion in the body}

To obtain the ECG, the electric potential has to be computed on the surface of the body. For that purpose, the electrostatic equation satisfied by $\phi_{\mathrm{T}}$ is solved in $\Omega_{\mathrm{T}}$ :

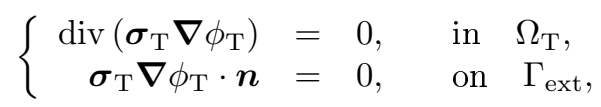

where the conductivity $\boldsymbol{\sigma}_{\mathrm{T}}$ takes different values in the different regions of the torso (see Table A3).

\subsection{Coupling the three models together}

To close the system, the continuity of the potential and the normal current are imposed at the interfaces between the aorta and the torso $\partial \Omega_{\mathrm{A}}$ and between the heart and the torso $\partial \Omega_{\mathrm{H}}$

$$
\left\{\begin{array} { r l } 
{ \boldsymbol { \sigma } _ { \mathrm { bl } } \boldsymbol { \nabla } \phi _ { \mathrm { A } } \cdot \boldsymbol { n } } & { = \boldsymbol { \sigma } _ { \mathrm { T } } \boldsymbol { \nabla } \phi _ { \mathrm { T } } \cdot \boldsymbol { n } , } \\
{ \phi _ { \mathrm { A } } } & { = \phi _ { \mathrm { T } } , }
\end{array} \quad \text { and } \quad \left\{\begin{array}{rl}
\boldsymbol{\sigma}_{\mathrm{e}} \boldsymbol{\nabla} \phi_{\mathrm{H}} \cdot \boldsymbol{n} & =\boldsymbol{\sigma}_{\mathrm{T}} \boldsymbol{\nabla} \phi_{\mathrm{T}} \cdot \boldsymbol{n}, \\
\phi_{\mathrm{H}} & =\phi_{\mathrm{T}} .
\end{array}\right.\right.
$$

Equations (4), (5) and (7), supplemented with the fluid boundary conditions and (6), are strongly coupled together by the transmission conditions (8). This makes the global model particularly challenging from a scientific computing standpoint.

Remark 2.1 In the literature, it is sometimes assumed that the extracellular medium is isolated, i.e. $\boldsymbol{\sigma}_{\mathrm{e}} \boldsymbol{\nabla} \phi_{\mathrm{H}} \cdot \boldsymbol{n}=0$ on $\partial \Omega_{\mathrm{H}}$. This assumption considerably simplifies the problem since it decouples the heart and the torso. But it does, however, have some impact on the ECG [3]. In particular, it significantly increases the amplitude of the ECG. Since our goal is to capture the modification of the amplitude of the signal, we wish to avoid a priori simplifications that might affect the ECGs.

\section{Numerical methods}

All the equations are discretized with P1 finite elements. This section is devoted to a brief presentation of the time schemes and the coupling algorithm. Let $N$ be a positive integer, $T>0$ the final time and $\tau=T / N$ the time step. Given the initial conditions, the discrete solutions $\boldsymbol{u}_{h}^{n}$, $p_{h}^{n}$ and $\phi_{\mathrm{A}, h}^{n}$ are computed in the aorta, $V_{\mathrm{m}, h}^{n}$ and $\phi_{\mathrm{H}, h}^{n}$ in the heart and $\phi_{\mathrm{T}, h}^{n}$ in the torso, for $n=1, \ldots, N$.

The MHD model of the aorta (4) is solved with a fractional step method: given $\boldsymbol{u}_{h}^{n}, p_{h}^{n}$ and $\phi_{\mathrm{A}, h}^{n}$, compute $\left(\boldsymbol{u}_{h}^{n+1}, p_{h}^{n+1}, \phi_{\mathrm{A}, h}^{n+1}\right)$ in $\Omega_{\mathrm{A}}$ such that

$$
\left\{\begin{aligned}
\rho\left(\frac{\boldsymbol{u}_{h}^{n+1}-\boldsymbol{u}_{h}^{n}}{\tau}+\boldsymbol{u}_{h}^{n} \cdot \boldsymbol{\nabla} \boldsymbol{u}_{h}^{n+1}\right)-\eta \Delta \boldsymbol{u}_{h}^{n+1}-\boldsymbol{\sigma}_{\mathrm{bl}}\left(\boldsymbol{u}_{h}^{n+1} \times \boldsymbol{B}\right) \times \boldsymbol{B} & =-\boldsymbol{\nabla} p_{h}^{n}-\boldsymbol{\sigma}_{\mathrm{bl}} \boldsymbol{\nabla} \phi_{\mathrm{A}, h}^{n} \times \boldsymbol{B}, \\
\Delta p_{h}^{n+1} & =\frac{\rho}{\tau} \operatorname{div} \boldsymbol{u}_{h}^{n+1}, \\
\operatorname{div}\left(\boldsymbol{\sigma}_{\mathrm{bl}} \boldsymbol{\nabla} \phi_{\mathrm{A}, h}^{n+1}\right) & =\operatorname{div}\left(\boldsymbol{\sigma}_{\mathrm{bl}} \boldsymbol{u}_{h}^{n+1} \times \boldsymbol{B}\right) .
\end{aligned}\right.
$$

The advection is stabilized with a streamline diffusion term. In $(9)_{1}$, the pressure and the potential are treated explicitly, thus making it possible to solve $(9)_{1}$, and then $(9)_{2}$ and $(9)_{3}$ separately. 
During this study, implicit monolithic schemes were also considered. But with the parameters of blood flows, and with the time step needed for the bidomain equations, algorithm (9) proves to be stable and accurate. Since it is by far the most efficient, it is the algorithm used in the following.

The electrical heart model (5) is discretized in time by combining a second order Backward Differentiation Formula (BDF2) implicit scheme with an explicit treatment of the ionic current, as proposed in [3].

The interface conditions (8) strongly couple equations (4), (5) and (7) together. This coupling is performed using a Dirichlet-Neumann domain decomposition algorithm, the interface linear problem being solved by a matrix-free GMRES algorithm. The matrix-vector product of the interface problem is computed as follows: at each time step, the potentials coming from the aorta and the heart are sent to the torso where a Dirichlet problem is solved; then the torso sends back the variational form of the electrical current to the aorta and to the heart where a Neumann problem is solved; these iterations are repeated until convergence before the next time step. The "weak" coupling strategy essentially consists in doing only one iteration of the domain decomposition algorithm, starting with the homogeneous Neumann problems in the aorta and the heart.

\section{Numerical results}

\subsection{Verification tests}

In this section, we consider some tests to verify our MHD solver, the validity of the inductionless approximation of the MHD equations in blood flow regime and the coupling strategy.

4.1.1. Comparison with an analytical solution: pseudo-Hartmann 2d flow We consider the stationary counterpart of problem (4) over the $2 \mathrm{D}$ domain $\Omega=\left[0, L_{x}=1\right] \times\left[0, L_{y}\right]$ (Fig. 2, Left). The static magnetic field is $\boldsymbol{B}=\left[0,0, B_{0}\right]^{\top}$. A no-slip condition is imposed on $y=0$ and $y=L_{y}$, while a pressure gradient $G$ is imposed between the inlet and the outlet.
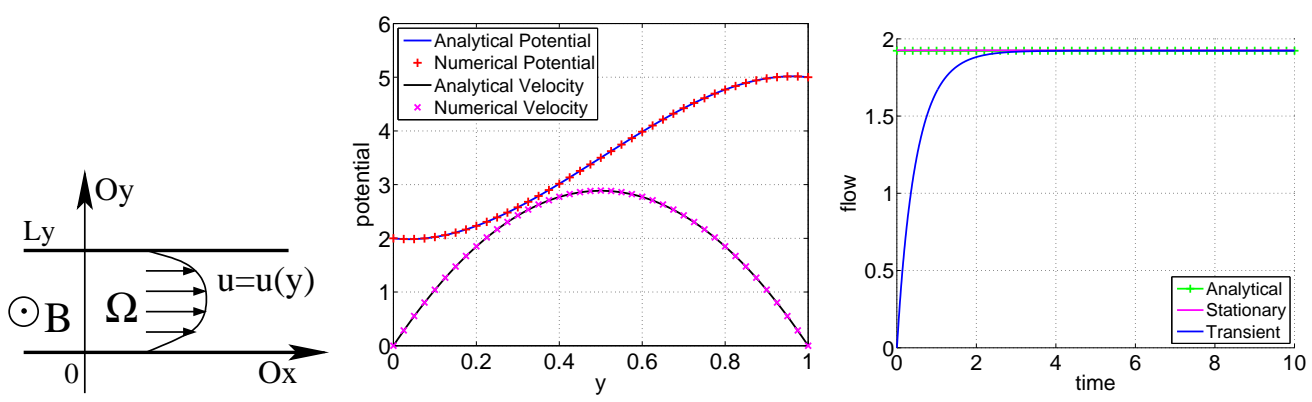

Figure 2. Left: the configuration. Middle: velocity (parabola) and potential as a function of $y$; analytical (solid line) and numerical (crosses) solutions. Right: inlet flux as a function of time, comparison between stationary analytical solution and numerical solutions.

This configuration is similar to a Hartmann flow [11, 22], however it differs in two ways. Firstly, the standard Hartmann solutions usually refer to the original MHD equations (3), whereas its inductionless approximation (4) is considered here. Secondly, the static magnetic field $\boldsymbol{B}$ is not imposed along the usual direction $O y$ but along $O z$, in order to avoid trivial uncoupling. 
The velocity can be written as $\boldsymbol{u}=[u(y), 0,0]^{\top}$, while the pressure is linear in $x, p=-G x$, and the electric potential only depends on $y, \phi=\phi(y)$. Thus system (4) is reduced to

$$
\begin{aligned}
-\eta u^{\prime \prime}(y)+\sigma B_{0}^{2} u(y)-G & =-\sigma B_{0} \phi^{\prime}(y) \\
\phi^{\prime \prime}(y) & =-B_{0} u^{\prime}(y) .
\end{aligned}
$$

Solving (10), we obtain $u=C y\left(L_{y}-y\right)$, and $\phi=\frac{C B_{0}}{3} y^{3}-\frac{C B_{0} L_{y}}{2} y^{2}+\frac{G-2 C \eta}{\sigma B_{0}} y+\phi_{0}$, where $C$ and $\phi_{0}$ are fixed by the boundary conditions. Two cases are considered.

Imposed potentials Assuming that $\phi(0)=\phi_{0}$ and $\phi\left(L_{y}\right)=\phi_{0}+\delta \phi$, with given constants $\phi_{0}$ and $\delta \phi$, we obtain $C=\frac{6}{\eta} \frac{G-\sigma B_{0} \delta \phi / L_{y}}{12+(\sigma / \eta) B_{0}^{2} L_{y}^{2}}$. Fig. 2 shows the very good agreement of the numerical result and this analytical solution. The parameters are $B_{0}=-2, \sigma=0.5, \eta=0.05, \phi_{0}=2, \delta \phi=3, G=$ $2, L_{y}=1, H_{y}=7, \tau=10^{-2}$ ( $S I$ units, arbitrary values).

Coupling with a Poisson equation To verify the multi-domain coupling algorithm, we supplement $\Omega$ with the domain $D=\left[0, L_{x}\right] \times\left[L_{y}, H_{y}\right],\left(H_{y}>L_{y}\right)$ where no fluid flows, but where the potential satisfies a Poisson equation (Fig. 3). This configuration roughly mimics the aorta and the torso.
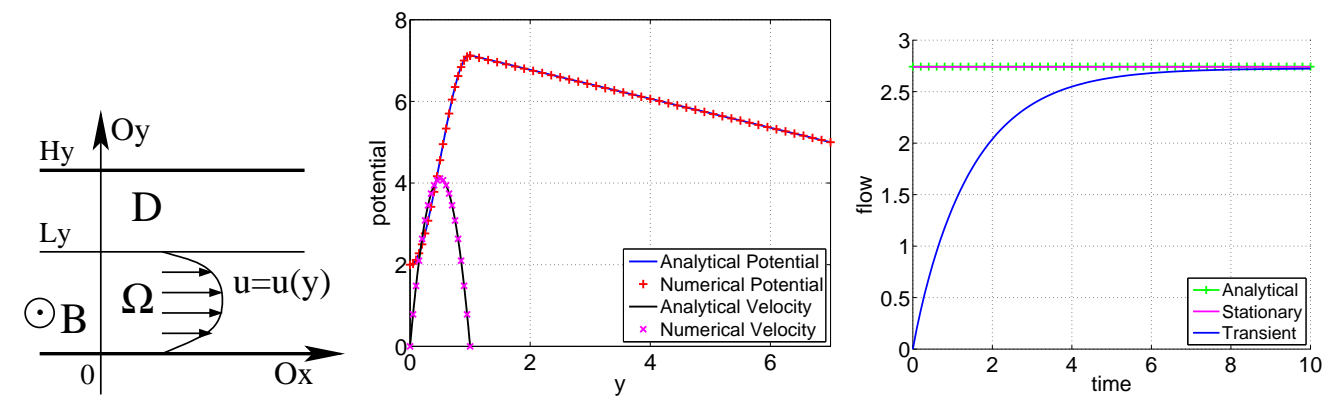

Figure 3. Left: the configuration. Middle: velocity (parabola) and potential as a function of $y$; analytical (solid line) and numerical (crosses) solutions. Right: inlet flux as a function of time, stationary analytical solution and numerical solutions.

In $D$, the potential satisfies $-\operatorname{div}(\sigma \nabla \phi)=0$. With the boundary conditions $\phi(0)=\phi_{0}$ and $\phi\left(H_{y}\right)=\phi_{0}+\delta \phi$ (where $\phi_{0}$ and $\delta \phi$ are two given constants) and using the transmission conditions (8) at $y=L_{y}$, we obtain $C=\frac{6}{\eta} \frac{G-\sigma B_{0} \delta \phi / H_{y}}{12+(\sigma / \eta) B_{0}^{2} L_{y}^{3} / H_{y}}$ and

$$
\phi= \begin{cases}C B_{0} \frac{y^{3}}{3}-C B_{0} L_{y} \frac{y^{2}}{2}+\frac{G-2 C \eta}{\sigma B_{0}} y+\phi_{0}, & y \leq L_{y} \\ \frac{G-2 C \eta}{\sigma B_{0}}\left(y-H_{y}\right)+\phi_{0}+\delta \phi, & y \geq L_{y} .\end{cases}
$$

Again, the analytical and the numerical solutions are in very good agreement (Fig. 3).

4.1.2. Comparison with an analytical solution: non-conducting straight tube Gold [12] provides the solution to the classical MHD (3) in the case of a steady flow of an incompressible, viscous, electrically conducting fluid through a insulating circular pipe in the presence of a transverse uniform magnetic field. The analytical solution (mean flow, velocity, electric potential along a diameter) is 
computed as a series of modified Bessel functions of the first kind [1]. The analytical solutions is approximated by truncated series.

For comparison, we solve the inductionless MHD (4) numerically in a physical configuration close to the blood flows. The domain $\Omega$ is a straight cylinder of radius $R=1 \mathrm{~cm}$ and oriented along $O z$. The viscosity is $\eta=0.05$ Poise and the conductivity is $\sigma=5 \cdot 10^{-3} \mathrm{Scm}^{-1}$. A pressure gradient $G=-\frac{d p}{d z}=24 \mathrm{~g} \mathrm{~cm}^{-2} \mathrm{~s}^{-2}$ is imposed between the inlet and the outlet. A static magnetic field of $0,10,100 T$ is imposed along $O x$. The wall is insulating and the potential does not depend on $z$. The boundary conditions are thus

$$
\left\{\begin{array} { r l } 
{ p ( z = L ) = L G } & { \text { on } \Gamma _ { L } , } \\
{ p ( z = 0 ) = 0 } & { \text { on } \Gamma _ { 0 } , }
\end{array} \quad \text { and } \quad \left\{\begin{array}{rl}
\boldsymbol{u}=0 & \text { on } \Gamma_{\text {wall }} \\
\nabla \phi \cdot \boldsymbol{n}=0 & \text { on } \partial \Omega .
\end{array}\right.\right.
$$

Three different levels of mesh refinement were considered, denoted by $M_{i, i=1,2,3}$ containing respectively 88000,380000 and 1500000 tetrahedra. Table 1 shows the flux, the maximum axial velocity, the transverse difference of electric potential and the maximum electrical current modulus. The impact of the magnetic field on the flow is very small (about $2 \%$ of flow reduction between $\boldsymbol{B}=0 T$ and $\boldsymbol{B}=10 T$ ). Fig. 4 and 5 show the very good agreement of the velocity, the potential and the electrical current with the analytical solution. These results confirm the validity of the inductionless approximation for MHD in blood flows with $\boldsymbol{B}$ ranging from 0 to 100T. Abi-Abdallah et al [1] also reached the same conclusion.

Transient simulations starting from 0 are performed on the mesh $M_{2}$. In Fig. 6 , the maximum velocity and the potential are represented as a function of time for $\boldsymbol{B}=0,10$, and $100 \mathrm{~T}$. The stationary analytical solutions are also reported. As expected, the transient values actually tend to the correct numerical stationary state.

\begin{tabular}{c|c|c|c|c|c|c}
\hline & $B(T)$ & $H a$ & Flux $\left(\mathrm{cm}^{3} \mathrm{~s}^{-1}\right)$ & $V_{\max }\left(\mathrm{cm} \mathrm{s}^{-1}\right)$ & $\Delta \phi(m V)$ & $\|\boldsymbol{j}\| \|^{\max }\left(A \mathrm{~m}^{-2}\right)$ \\
\hline exact MHD & 0 & 0 & 188.496 & 120 & 0 & 0 \\
inductionless, $M_{1}$ & 0 & 0 & 187.08 & 119.37 & 0 & 0 \\
inductionless, $M_{2}$ & 0 & 0 & 188.02 & 119.79 & 0 & 0 \\
inductionless, $M_{3}$ & 0 & 0 & 188.29 & 119.91 & 0 & 0 \\
\hline exact MHD & 10 & 1 & 184.706 & 116.400 & 117.389 & 2.9546 \\
inductionless, $M_{1}$ & 10 & 1 & 183.30 & 115.79 & 117.01 & 2.973 \\
inductionless, $M_{2}$ & 10 & 1 & 184.24 & 116.20 & 117.30 & 2.970 \\
inductionless, $M_{3}$ & 10 & 1 & 184.50 & 116.31 & 117.36 & 2.965 \\
\hline exact MHD & 20 & 2 & 174.758 & 107.134 & 221.250 & 5.6678 \\
inductionless, $M_{1}$ & 20 & 2 & 173.38 & 106.56 & 220.36 & 5.698 \\
inductionless, $M_{2}$ & 20 & 2 & 174.30 & 106.94 & 220.94 & 5.695 \\
inductionless, $M_{3}$ & 20 & 2 & 174.56 & 107.05 & 221.06 & 5.688 \\
\hline exact MHD & 100 & 10 & 90.634 & 42.882 & 550.171 & 16.6321 \\
inductionless, $M_{1}$ & 100 & 10 & 89.02 & 42.21 & 542.99 & 16.45 \\
inductionless, $M_{2}$ & 100 & 10 & 90.06 & 42.65 & 547.75 & 16.61 \\
inductionless, $M_{3}$ & 100 & 10 & 90.39 & 42.78 & 549.22 & 16.65 \\
\hline
\end{tabular}

Table 1. Gold validation: analytical and numerical results on various mesh refinements. The dimensionless Hartmann number is defined as $H a=\boldsymbol{B} R \sqrt{\sigma / \eta}(=0.1 \boldsymbol{B}$ here $)$.

4.1.3. Comparison with a numerical solution from the literature In [16], a 2D model was proposed to study the electric potential induced by the MHD effects in the aorta. This test case cannot be used for quantitative estimations of the MHD effect in MRI because the magnetic field $\boldsymbol{B}$ is imposed along the back-front axis and not along the feet-head axis. Nevertheless, for validation 

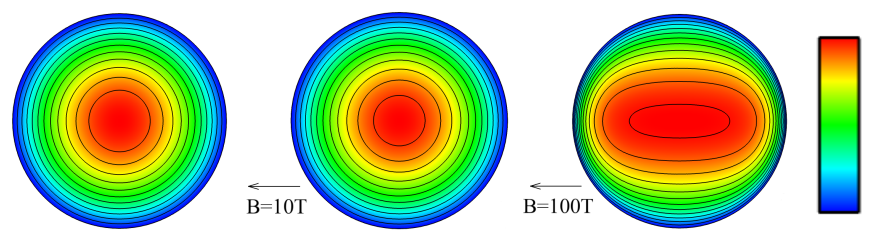

Figure 4. Gold validation: velocity contours. Left: $\boldsymbol{B}=0 T$, colorbar scale $[0,119.9] \mathrm{cm} \mathrm{s}^{-1}$. Center: $\boldsymbol{B}=10 \mathrm{~T}$, scale $[0,116.3] \mathrm{cm} \mathrm{s}^{-1}$. Right: $\boldsymbol{B}=100 \mathrm{~T}$, scale $[0,42.6] \mathrm{cm} \mathrm{s}^{-1}$.
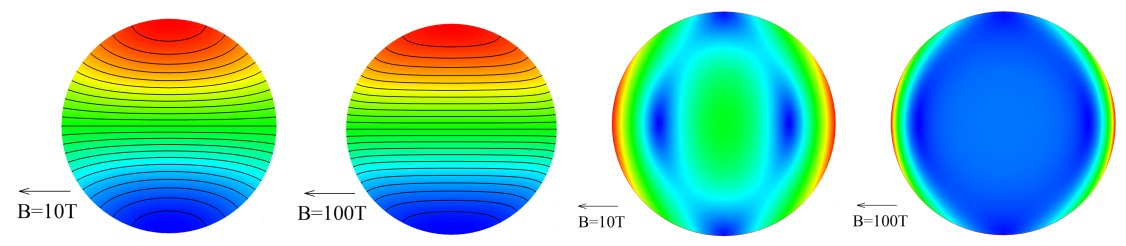

Figure 5. Gold validation: potential and electrical current on a cut plane. Left: potential, $\boldsymbol{B}=10 \mathrm{~T}$, scale $[-58.6,58.6] \mathrm{mV}$. Middle left: potential, $\boldsymbol{B}=100 \mathrm{~T}$, scale [-274, 274] $\mathrm{mV}$. Middle right: current, $\boldsymbol{B}=10 \mathrm{~T}$, scale $[0,2.97] \mathrm{A} \mathrm{m}^{-2}$. Right: current, $\boldsymbol{B}=100 \mathrm{~T}$, scale $[0,16.61] \mathrm{A} \mathrm{m}^{-2}$.
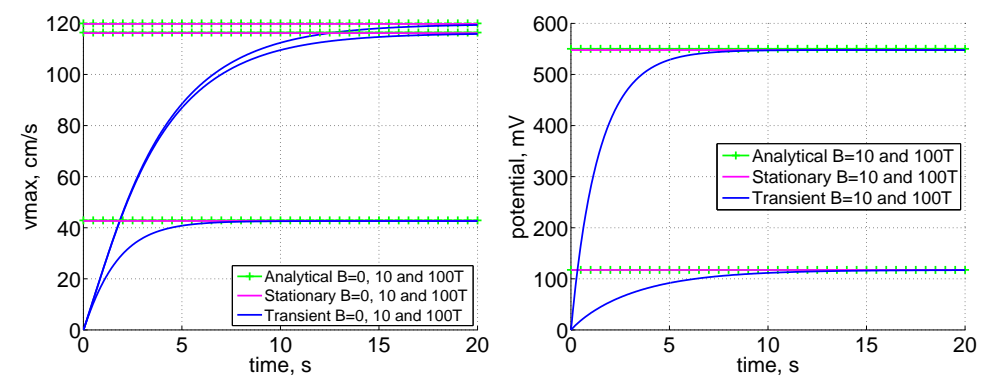

Figure 6. Gold validation. Maximum velocity and potential versus time. Convergence of the transient numerical solutions to the analytical stationary ones. Left: $\boldsymbol{B}=0 T$ (top curves), $\boldsymbol{B}=10 T$ and $\boldsymbol{B}=100 T$ (bottom curves). Right: $\boldsymbol{B}=10 T$ (bottom) and $\boldsymbol{B}=100 T$ (top).

purposes, we compared the results from [16] with those given by the solution of the 3D MHD model (4) coupled to the diffusion in the torso (7), and we obtained identical results, see [18].

\section{2. $M H D$ effects on the $E C G$}

This section contains our main results. The three models - MHD in the aorta (4), bidomain in the heart (5) and diffusion in the torso (7) - are coupled together and solved on a realistic anatomy. The body mesh contains three regions with different conductivities: bones in the torso, lungs and remaining parts. A heart, limited to two ventricles, and a large portion of an aorta are inserted into the body. The aorta comes from [21], all the other parts come from Zygote Media Group, Inc. The three subdomains - aorta, heart, body — were meshed using ghs3d [10], see Table 2 and Fig. 7. The mesh of the heart was chosen so as to be fine enough to capture the front propagation (refinement intermediate between R2 and R3 of Table 5 in [3]). Mesh convergence was checked on the ECG: less than $2 \%$ change in the peak value of the ECG was observed with more refined 
meshes, see Fig. 8 right. The time step is $\tau=0.25 \mathrm{~ms}$ for all computations in order to model the electrophysiology accurately. As observed in [3], larger time steps produce undesirable spurious oscillations during the $Q R S$ complex. This time step was checked to be small enough for the MHD. The computation was performed over 3 cardiac cycles. The data used for the computations are given in Tables A1-A3. The conductivities are taken from [26]. The blood in the ventricles is assumed to be still and is treated as a passive diffusive domain.

The ECGs are obtained by plotting linear combinations of the values of $\phi_{\mathrm{T}}$ on 9 standard points of the body surfaces (Fig. 7 right). See [17] p. 277 for a definition of these quantities. The ECGs obtained without MHD $(\boldsymbol{B}=0 T)$ are considered a good reference to evaluate the impact of MHD. Various improvements could be made in the ECG simulation. In spite of the flaws observed in this simulated ECG (see e.g. the discussion in [3]), we believe that it can be considered reasonable for the purpose of this paper, which is to study the perturbations of the ECG by the MHD effect.

\begin{tabular}{rrrc}
\hline Mesh & \# vertices & \# tetrahedra & mesh quality $\left(Q_{m} \leq 10\right)$ \\
\hline heart & 108112 & 541994 & $100 \%$ \\
aorta & 22798 & 110362 & $100 \%$ \\
body & 264010 & 1437391 & $99.95 \%$ \\
\hline
\end{tabular}

Table 2. Meshes for the heart, the aorta and the rest of the body. Mesh quality of a tetrahedron defined as: $Q_{m}=\frac{1}{6 \sqrt{6}} h_{\max } \frac{\sum_{i=1}^{4} \text { area(faces) }}{\text { volume }}(\geq 1)$. We give the percentage of tetrahedra having a good quality $\left(Q_{m} \leq 10\right)$.
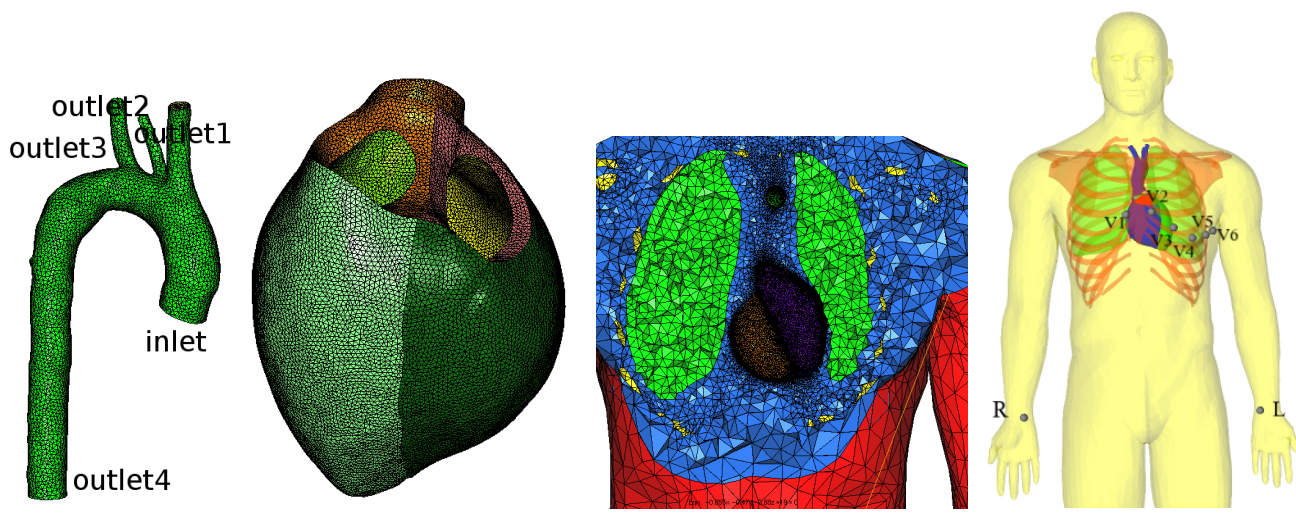

Figure 7. Meshes of the three domains: aorta, heart and body with 3 different tissues (bones, lungs, other tissues), body surface with some points used to compute the ECG.

4.2.1. Electrocardiograms with $M H D$ effects In Fig. 8 (left), we show the flow $Q$ imposed at the inlet of the aorta. We also show the ECG in the same picture, in order to show how the velocity pulse is located in time with respect to the cardiac cycle: we set the pulse so that the ejection of blood in the aorta immediately follows the $Q R S$ complex (taken from [6]). The $T$-wave occurs at approximately $t \approx 250 \mathrm{~ms}$.

The 12-lead ECGs represented in Fig. 9 were obtained by solving the full coupled problem with $B=0,1,2,3,5,8$ and $10 T$. With $B=0$, the results are similar to those presented in [3, 4]: 

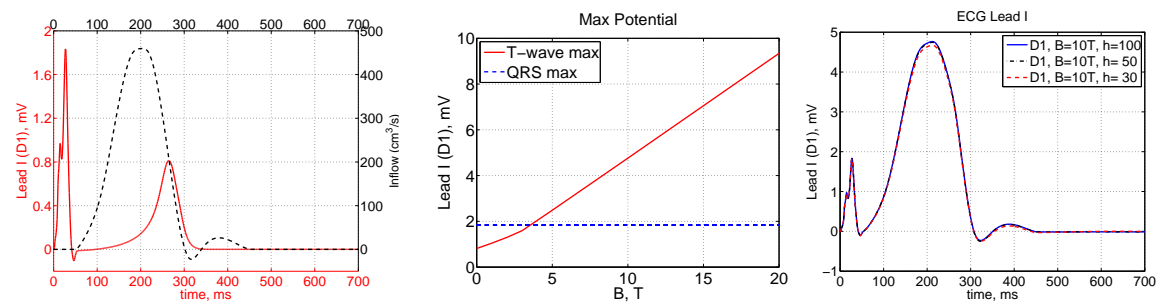

Figure 8. Left: lead $I$ of the ECG with $\boldsymbol{B}=0$, in $m V$ (solid line) and imposed inlet flow $Q$ (dashed-line) in $\mathrm{cm}^{3} \mathrm{~s}^{-1}$, as a function of time $(\mathrm{ms})$. The total flux is approximately $5 \mathrm{~L} \mathrm{~min}^{-1}$. Middle: maximum potential value for the lead $I(m V)$ as a function of $\boldsymbol{B}(T)$. The peak value in $Q R S$ complex is shown (horizontal broken line, constant for all $B$ ). Right: lead $I$ (" $D 1$ ") of the ECG with $\boldsymbol{B}=10 T$, in $m V$, for 3 mesh refinements. The 3 curves are almost superimposed.

the peak during the $Q R S$ complex is approximately $1.8 \mathrm{mV}$ for the lead $I$, and the peak during the $T$-wave reaches approximately $0.8 \mathrm{mV}$. The precordial leads $V_{3}, V_{4}$ and $V_{5}$ are almost unaffected by the magnetic field. In all other leads, the $T$-wave is altered by the MHD in the aorta, and this effect is amplified when $\boldsymbol{B}$ increases. The MHD effect is particularly strong for the limb leads $I, I I$, $I I I$, the augmented limb leads $a V R, a V L, a V F$ and for the precordial lead $V_{1}$. In Table 3 , we report for various $\boldsymbol{B}$ the extremal values of the electric potential in the aorta and on the body surface, the peak values of the leads $I, I I$ and $I I I$, and the maximum electrical current intensity in the aorta. In lead $I$ (Fig. 9, top left), the $T$-wave peak increases from $0.8 \mathrm{mV}$ when $\boldsymbol{B}=0 T$, to $1.6 \mathrm{mV}$ when $\boldsymbol{B}=3 T$, and up to $3.85 \mathrm{mV}$ when $\boldsymbol{B}=8 T$. Leads $I I$ and $I I I$ are less affected. The maximum value increases quasi-linearly with $\boldsymbol{B}$, see Fig. 8, right. These results are in relatively good agreement with those of [13], although the ECG given in their Fig.1 shows a more pronounced MHD effect than ours for lead II. Different experimental values are reported in the literature for the $T$-wave elevation during MRI examinations: Nijm et al [23] measured a $T$-wave elevation of order $0.2 \mathrm{mV}$ for healthy subjects inside a $3 T$ MRI scanner. Abi-Abdallah et al [2] found increases of between 0.2 and $0.7 \mathrm{mV}$ (about $300 \%$ increase) for subjects exposed to a 1.5 Tesla magnet.

\begin{tabular}{c|cc|ccc|ccc|ccc|c}
\hline Wave & $\begin{array}{c}\boldsymbol{B} \\
(T)\end{array}$ & $H a$ & $\phi_{\mathrm{A}}^{\min }$ & $\begin{array}{c}\phi_{\mathrm{A}}^{\max } \\
(\mathrm{m})\end{array}$ & $\Delta \phi_{\mathrm{A}}$ & $\phi_{\mathrm{S}}^{\min }$ & $\begin{array}{c}\phi_{\mathrm{S}}^{\max } \\
(m V)\end{array}$ & $\Delta \phi_{\mathrm{S}}$ & Lead $I$ & $\begin{array}{c}\text { Lead II } \\
(m V)\end{array}$ & Lead $I I I$ & $\begin{array}{c}\left\|\boldsymbol{j}_{A}\right\|^{\max } \\
\left(A m^{-2}\right)\end{array}$ \\
\hline$R$ & \multicolumn{2}{|c}{ all } & -4.9 & 1.9 & 6.8 & -6.3 & 2.0 & 8.3 & 1.83 & 1.47 & 0.66 & 0.23 \\
\hline & 0 & 0 & -0.5 & -0.02 & 0.48 & -0.06 & 0.5 & 0.56 & 0.81 & 0.42 & -0.40 & 0.039 \\
& 1 & 0.12 & -9.7 & 8.5 & 18.2 & -1.0 & 1.23 & 2.23 & 1.05 & 0.49 & -0.57 & 0.61 \\
$T-$ & 2 & 0.24 & -19.5 & 17 & 36.5 & -2.0 & 2.35 & 4.35 & 1.30 & 0.56 & -0.76 & 1.22 \\
wave & 3 & 0.36 & -29 & 25.6 & 54.6 & -3.0 & 3.33 & 6.33 & 1.59 & 0.64 & -1.02 & 1.84 \\
& 5 & 0.59 & -49 & 43 & 92 & -4.9 & 5.7 & 10.6 & 2.49 & 0.87 & -1.61 & 3.06 \\
& 8 & 0.95 & -78 & 68 & 146 & -8.1 & 8.85 & 16.9 & 3.85 & 1.35 & -2.50 & 4.90 \\
& 10 & 1.19 & -97 & 86 & 183 & -9.8 & 11 & 20.8 & 4.76 & 1.67 & -3.10 & 6.13 \\
& 15 & 1.78 & -146 & 128 & 274 & -15 & 16.6 & 31.6 & 7.05 & 2.47 & -4.58 & 9.19 \\
& 20 & 2.38 & -195 & 171 & 366 & -20 & 22 & 42 & 9.34 & 3.27 & -6.08 & 12.2 \\
\hline
\end{tabular}

Table 3. Potential and current as a function of $\boldsymbol{B}$ : maximum and minimum potentials in the aorta $\left(\phi_{\mathrm{A}}\right)$ and on the skin $\left(\phi_{\mathrm{S}}\right)$, peak value for leads $I, I I$ and $I I I$, peak current modulus in the aorta $\left(j_{A}\right)$. Values at $t \approx 210 \mathrm{~ms}$, peak value for the MHD effects ( $T$-wave), and value during the $R$-wave at $t=27 m s$ (it is identical for all $\boldsymbol{B}$ ). The Hartmann number is computed as $H a=B R_{A} \sqrt{\boldsymbol{\sigma}_{\mathrm{bl}} / \eta}$, see Table A2.

Fig. 9 shows that the ECG is roughly the superposition of the ECG without a magnetic field 

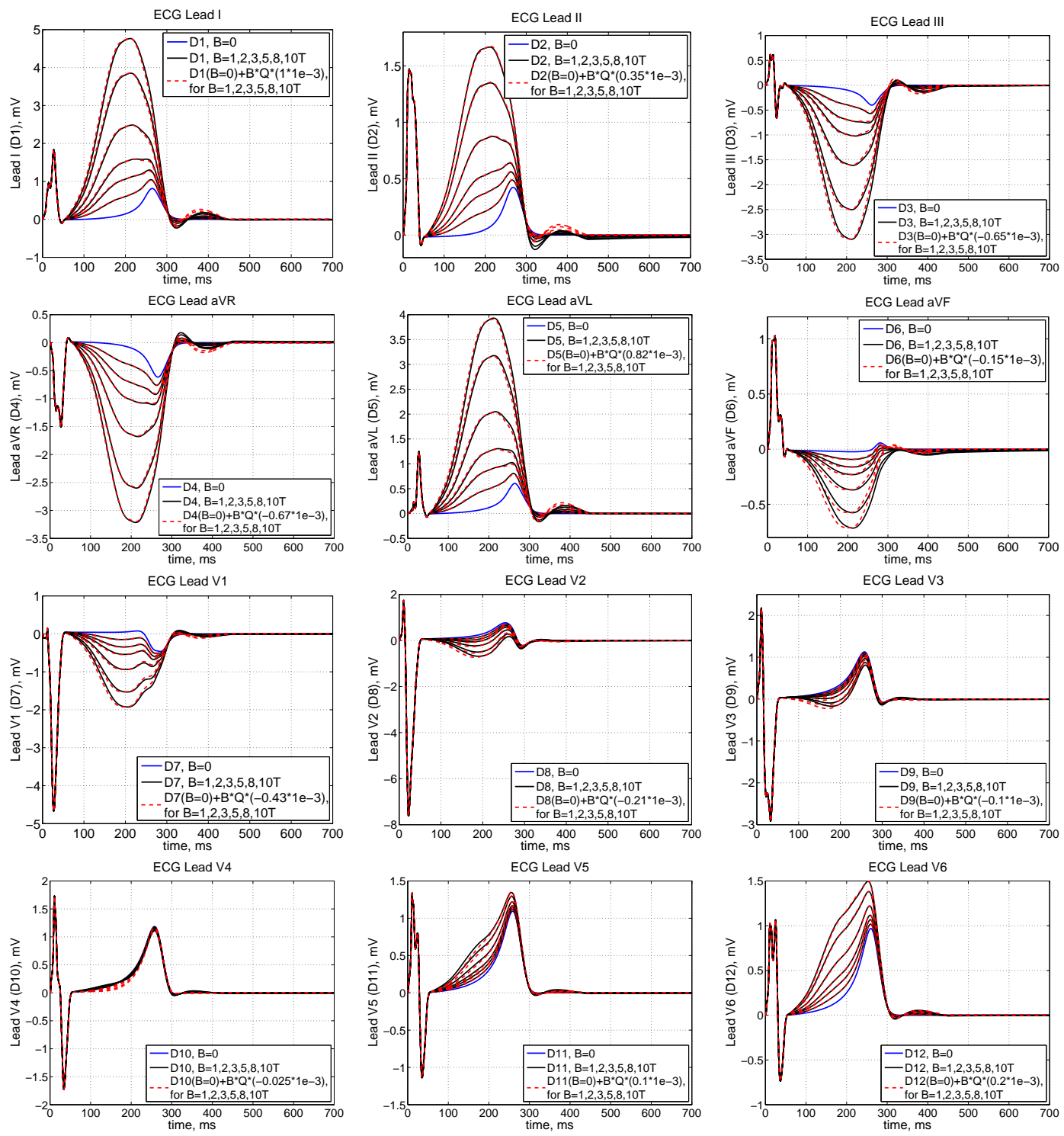

Figure 9. 12-leads $D_{i}(B)$ with MHD for various $B=0,1,2,3,5,8,10 T$. The scales vary in each case. The ECG are drawn with solid lines. The secondary peak increases with $\boldsymbol{B}$. The dashed lines represent the functions $D_{i}(B=0)+\alpha_{i} B Q\left(\mathrm{~cm}^{3} \mathrm{~s}^{-1}\right)$ (see (12)). 
and of a quantity proportional to the magnetic field $\boldsymbol{B}$ and to the aortic flux $Q$ :

$$
D_{i}(B) \approx D_{i}(B=0)+\alpha_{i} B Q \quad(m V),
$$

where $D_{i}$ is the $i^{t h}$ lead and the coefficients $\alpha_{i}, i=1, \ldots, 12$, are given in Table 4 . The equation (12) is a good approximation for almost all leads, except some precordial leads, particularly $V_{3}$ and $V_{4}$.

\begin{tabular}{c|c|c|c|c|c|c}
\hline & $I\left(D_{1}\right)$ & $I I\left(D_{2}\right)$ & $I I I\left(D_{3}\right)$ & $a V R\left(D_{4}\right)$ & $a V L\left(D_{5}\right)$ & $a V F\left(D_{6}\right)$ \\
\hline$\alpha_{i}$ & $110^{-3}$ & $0.3510^{-3}$ & $-0.6510^{-3}$ & $-0.6710^{-3}$ & $0.8210^{-3}$ & $-0.1510^{-3}$ \\
\hline & $V_{1}\left(D_{7}\right)$ & $V_{2}\left(D_{8}\right)$ & $V_{3}\left(D_{9}\right)$ & $V_{4}\left(D_{10}\right)$ & $V_{5}\left(D_{11}\right)$ & $V_{6}\left(D_{12}\right)$ \\
\hline$\alpha_{i}$ & $-0.4310^{-3}$ & $-0.2110^{-3}$ & $-0.1010^{-3}$ & $-0.02510^{-3}$ & $0.1010^{-3}$ & $0.2010^{-3}$ \\
\hline
\end{tabular}

Table 4. Proportionality coefficients in the numerical relation (12). $\alpha_{i}$ in $10 \mathrm{~cm}^{-1}=\mathrm{mm}^{-1}$.

It might be feared that the MHD effect in the aorta induces a perturbation of the electrical activity of the heart, and possibly lead to fibrillation. According to our computations, this does not happen, even for strong magnetic field: the extracellular potential in the myocardium is only slightly modified by the aortic electrical activity, see Fig. 10 .
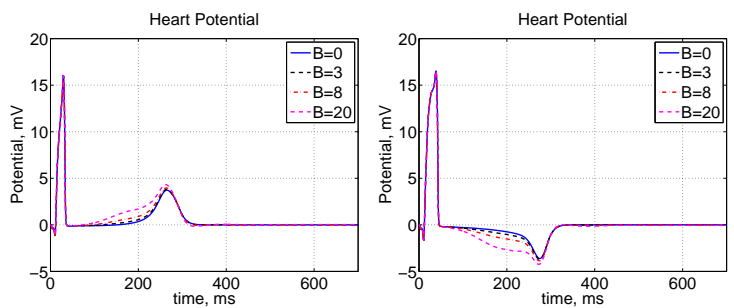

Figure 10. Potential $(m V)$ at two points on the heart surface (where the MHD effect is the strongest) as a function of time $(m s)$, for $\boldsymbol{B}=0,3,8,20 T$.

We also performed a computation using the weak coupling strategy, see Fig. 11 and Remark 2.1. This simplification results in a modification of the amplitude in the $Q R S$ complex and in the $T$ wave, but with no clear pattern, as it can either increase or decrease their relative amplitudes, see the leads $I$ and $I I$ in Fig. 11 for $B=10 T$. It is therefore questionable whether the weak coupling simplification is able to assess the MHD effects on the ECG satisfactorily.
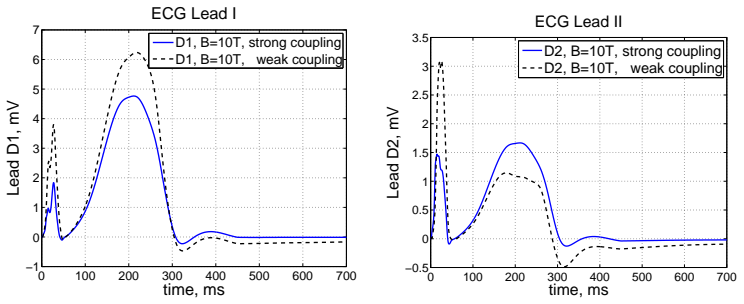

Figure 11. Leads $I$ (Left) and $I I$ (Right) with MHD effects $(\boldsymbol{B}=10 T)$. Comparison between the strong coupling strategy (solid line) and the weak coupling strategy (dashed line). 
4.2.2. MHD effect in the aorta and in the body In Fig. 12, the electric potential on the skin is represented at various time instants for $B=0,1,3,8 T$. One can see the effects of the electrical activity of the heart particularly during the $Q R S$ complex and the $T$-wave. The MHD effect in the aorta clearly modifies the potential around $t=200 \mathrm{~ms}$. The velocity inside the aorta is almost unaffected by the MHD effect, even for large magnetic fields [19]. This observation is consistent with the results of [1] and of Section 4.1.2. The electric potential on the boundary of the aorta is represented in Fig. 13 during the diastole $(t \approx 200 \mathrm{~ms})$. The heart activity slightly modifies the potential of the aorta (Fig. 13, left). The maximum voltage due to MHD appears, as expected, across the aortic arch, where the velocity is roughly orthogonal to the magnetic field. The predicted values are of the order of $10 \mathrm{mV}$ to $100 \mathrm{mV}$, which is in agreement with the calculations of [1] and of [16]. The electrical current is represented in Fig. 14 and its maximum value is reported in Table 3 for various $\boldsymbol{B}$. The current intensity is the largest at the top and at the bottom of the aortic arch, near the aortic wall. This is in agreement with the Gold simulations (Fig. 5). The maximum electrical current density in the aorta is approximately $0.6 \mathrm{~A} \mathrm{~m}^{-2}$ at $\boldsymbol{B}=1 \mathrm{~T}, 1.8 \mathrm{~A} \mathrm{~m}^{-2}$ at $3 T$, and $4.9 \mathrm{Am}^{-2}$ at $8 T$. This value is roughly twice as large as in the Gold test case (for $H a=1$ it is $\approx 5.1 \mathrm{Am}^{-2}$ in the aorta whereas it is $2.9 \mathrm{~A} \mathrm{~m}^{-2}$ in the ideal tube, see Table 1). This difference can be explained by a larger maximum velocity, a more complex geometry (non-infinite tube, outlets, etc.), and a coupling with the surrounding tissue (conducting walls). These values can also be compared to the current densities generated by the cardiac activity that reach approximately $1 \mathrm{Am}^{-2}$ inside the heart [29]. However, the induced current significantly decreases with the distance from a small region of the aortic arch. In $[16,29]$, the authors predicted a maximum current of $1.15 \mathrm{Am}^{-2}$ at $5 T, 2.20 \mathrm{~A} \mathrm{~m}^{-2}$ at $10 \mathrm{~T}$ and $3.20 \mathrm{~A} \mathrm{~m}^{-2}$ at $15 \mathrm{~T}$. These values are two to three times smaller than ours. Two factors can explain these differences. Firstly, as recalled in Section 4.1.3, the orientation of $\boldsymbol{B}$ in [16] is not along the main body axis. Secondly, the flow pattern obtained in our realistic aortic arch solving the nonlinear Navier-Stokes equations is of course very far from a steady state flow in an infinite cylinder. The induced electrical current has therefore a much more complicated behavior than in the simplified models.

\section{Conclusions}

A model made up of three components has been proposed to simulate the effects of MHD in the aorta and its impact on ECGs: electrophysiology in the heart, MHD in the aorta and electrostatics in the rest of the body. The main contributions of this article are 1. the use and the justification of the inductionless approximation of the MHD, which is more convenient in the blood flow regime than conventional MHD equations, 2. an efficient numerical scheme to solve the MHD equations in this regime, 3 . the definition of several benchmarks to verify the numerical procedures, and 4 . the full coupling of the three compartments that allowed us to study whether the current induced in the aorta provides a feedback on the heart.

Standard 12-lead ECGs have been computed with and without a magnetic field. A larger $T$ wave was obtained in the presence of a magnetic field, as expected. The electric potential and the electrical current in the aorta have been computed. According to these computations, we found that the ECG is approximately the superposition of the electrophysiology and of the MHD effects (see (12)). The impact of the aortic MHD effect on the electric activity of the heart has been evaluated and seems limited, even for strong magnetic fields.

Despite the complexity of the model, it still could be enriched. In addition to the usual questions of blood flow modelling (inlet flow, outlet boundary conditions, the length of the artery 


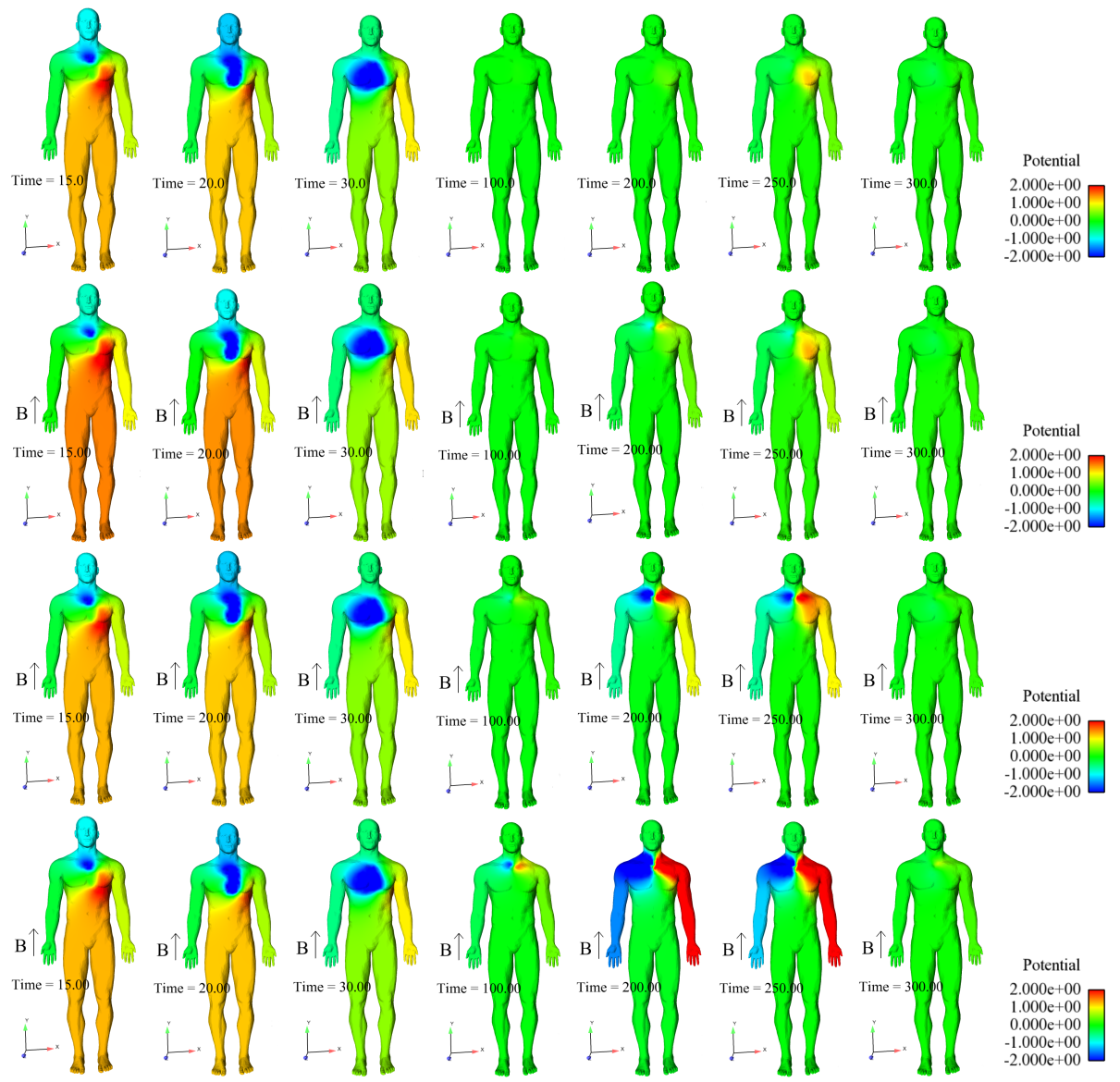

Figure 12. Electric potential. From top to bottom: $B=0 T$ (no MHD), $B=1,3,8 T$. From left to right, time instants: $t=15 \mathrm{~ms}, 20 \mathrm{~ms}, 30 \mathrm{~ms}, 100 \mathrm{~ms}, 200 \mathrm{~ms}, 250 \mathrm{~ms}, 300 \mathrm{~ms}$. Scale: $[-2,2] \mathrm{mV}$.

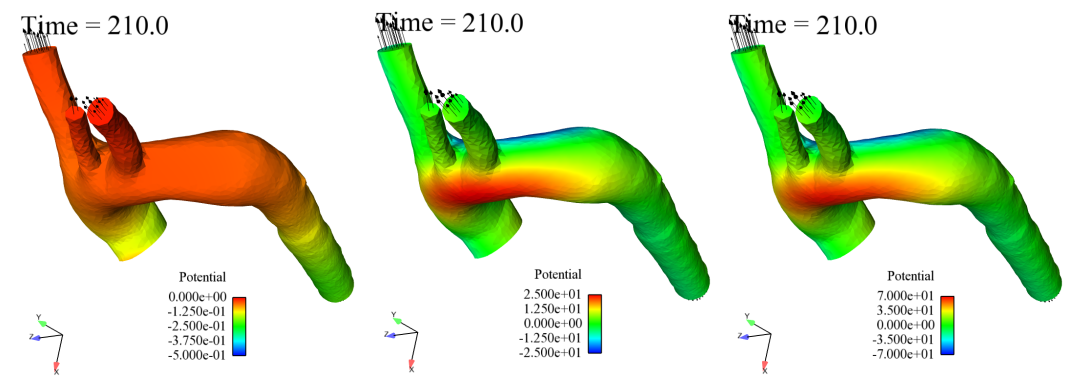

Figure 13. Electric potential on the aorta at $t=210 \mathrm{~ms}$ (systole): for $\boldsymbol{B}=0 T$ (left, scale: $[-0.5,0] \mathrm{mV}$ ), $\boldsymbol{B}=3 T$ (middle, scale: $[-25,25] \mathrm{mV}$ ) and $\boldsymbol{B}=8 T$ (right, scale: $[-70,70] \mathrm{mV}$ ). 


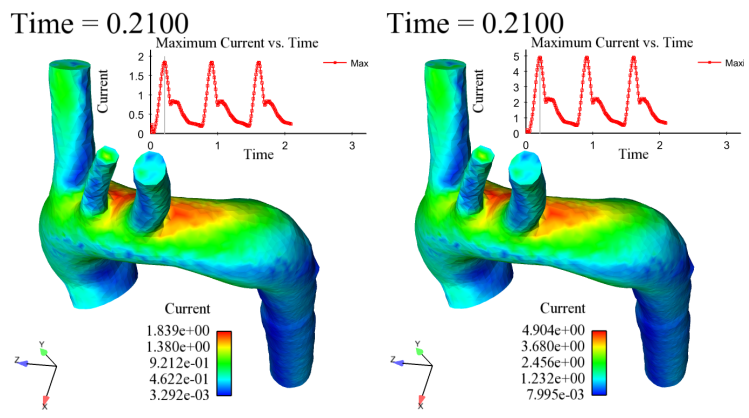

Figure 14. Electrical current intensity on the aorta at $t=210 \mathrm{~ms}$ (systole). Left: $\boldsymbol{B}=3 T$ (scale: $\left.[0,1.84] \mathrm{A} \mathrm{m}^{-2}\right)$. Right: $\boldsymbol{B}=8 T$ (scale: $\left.[0,4.9] \mathrm{A} \mathrm{m}^{-2}\right)$.

to be kept, etc.), several specific questions need to be discussed. For example, the blood flow in the pulmonary artery and in the heart might be also considered. We neglected these flows because we focused on the ECG, but they could have an effect on the cardiac electrophysiology. The elasticity of the aorta could be considered as in [21]. A major, but computationally expensive, improvement could be to link directly the aortic flow to an electromechanical model of the myocardium [4], and in this way the aortic inflow pulse would not be a given parameter of the model.

The data for the conductivities of different tissues used in the literature [25, 16] can vary by an order of magnitude. It could be interesting to perform a sensitivity analysis in order to evaluate the impact of these conductivities on the MHD effect. In the same direction, here we only took into account a few different tissue conductivities in the torso: it would also be interesting to explore a more precise description of the tissues (fat, muscles, more bones, etc.).

The aim of the present study was to demonstrate that the computational tool was able to capture a phenomenon that has actually been observed in MRI machines. In the future, the model could be used, for example to optimize the electrode locations so as to be less sensitive to the MHD effect, or on the contrary to extract more information from the MHD effect about the flow or the electrical properties of the tissues.

Acknowledgment: We wish to thank C.A. Taylor and C.A. Figueroa for the mesh of the aorta.

\section{Appendix A. Data values}

In this paper, the MHD computations in the aorta are performed using the $\{c m, g, s, m A\}$ system, that has the advantage of giving a convenient scaling for the blood flow, while preserving the magnetic field scaling $(1 T \longleftrightarrow 1\{\mathrm{~cm} / \mathrm{g} / \mathrm{s} / \mathrm{mA}\})$. In the results, the electric potential is given in $m V$ and the current in $A \mathrm{~m}^{-2}$. The computations of Section 4.2 use the following data. See Table A1 for the heart, Table A2 for the aorta, and Table A3 for the body.

[1] D. Abi-Abdallah, A. Drochon, V. Robin, and O. Fokapu. Effects of static magnetic field exposure on blood flow. Eur. Phys. J. Appl. Phys., 45(1):11301, 2009.

[2] D. Abi-Abdallah, V. Robin, A. Drochon, and O. Fokapu. Alterations in human ECG due to the magnetohydrodynamic effect: a method for accurate R peak detection in the presence of high MHD artifacts. In Conf Proc IEEE Eng Med Biol Soc., pages 1842-5, 2007.

[3] M. Boulakia, S. Cazeau, M.A. Fernández, J-F. Gerbeau, and N. Zemzemi. Mathematical modeling of electrocardiograms: A numerical study. Annals of Biomedical Engineering, 38:1071-1097, 2010.

[4] D. Chapelle, M. A. Fernández, J-F. Gerbeau, P. Moireau, J. Sainte-Marie, and N. Zemzemi. Numerical 


\begin{tabular}{llll}
\hline & Parameters & Value & units \\
\hline$T^{\star}$ & time period & 700 & $\mathrm{~ms}$ \\
$\sigma_{i, l}$ & intern longitudinal conductivity & $3.010^{-3}$ & $\mathrm{~S} \mathrm{~cm}-1$ \\
$\sigma_{i, t}$ & intern transverse conductivity & $3.010^{-4}$ & $\mathrm{~S} \mathrm{~cm}^{-1}$ \\
$\sigma_{e, l}$ & extern longitudinal conductivity & $3.010^{-3}$ & $\mathrm{~S} \mathrm{~cm}^{-1}$ \\
$\sigma_{e, t}$ & extern transverse conductivity & $1.210^{-3}$ & $\mathrm{~S} \mathrm{~cm}^{-1}$ \\
$A_{\mathrm{m}}$ & membrane area per vol & 200 & $\mathrm{~cm}^{-1}$ \\
$C_{\mathrm{m}}$ & membrane capacitance per area & $10^{-3}$ & $\mathrm{mF}$ \\
$\phi_{\mathrm{H}}$ & electric potential & $\approx 10$ & $\mathrm{mV}$ \\
\hline
\end{tabular}

Table A1. Main parameter values in the heart. See [3] for more details.

\begin{tabular}{lllll}
\hline & Parameters & Value & $\{\mathrm{cm} / \mathrm{g} / \mathrm{s} / \mathrm{mA}\}$ units & Value (SI unit) \\
\hline$T^{\star}$ & time period & 0.7 & $\mathrm{~s}$ & $9.510^{-3} \mathrm{~m}$ \\
$R_{A}$ & aortic arch radius & 0.95 & $\mathrm{~cm}$ & $1.0610^{3} \mathrm{~kg} \mathrm{~m}^{-3}$ \\
$\rho$ & blood density & 1.06 & $\mathrm{~g} \mathrm{~cm} \mathrm{~cm}^{-3}$ & $410^{-3} \mathrm{~kg} \mathrm{~m}^{-1} \mathrm{~s}^{-1}$ \\
$\eta$ & blood viscosity & 0.04 & $\mathrm{~g} \mathrm{~cm} \mathrm{~cm}^{-1} \mathrm{~s}^{-1}$ & $0.625 \mathrm{Sm}$ \\
$\boldsymbol{\sigma}_{\mathrm{bl}}$ & blood conductivity & $6.2510^{-4}$ & $\mathrm{~mA}^{2} \mathrm{~s}^{3} \mathrm{~g}^{-1} \mathrm{~cm}^{-3}$ & 1 to $20 \mathrm{~T}$ \\
$\boldsymbol{B}$ & magnetic field & 1 to 20 & $\mathrm{~g} \mathrm{~s}^{-2} \mathrm{~mA}^{-1}$ & $0.98 \mathrm{~m} \mathrm{~s}^{-1}$ \\
$\bar{u}_{\max }$ & mean inlet velocity & 98 & $\mathrm{~cm} \mathrm{~s}^{-1}$ & $0.4610^{-3} \mathrm{~m}^{3} \mathrm{~s}^{-1}$ \\
$q_{\max }$ & max blood flow & 460 & $\mathrm{~cm}^{3} \mathrm{~s}^{-1}$ & $4.6610^{-4} \mathrm{~m}^{2}$ \\
$S$ & inlet area & 4.66 & $\mathrm{~cm}^{2}$ & \\
$Q$ & blood flow rate & $\approx 5.36$ & $\mathrm{~L} \mathrm{~min}^{-1}$ & \\
$\phi_{\mathrm{A}}$ & electric potential & $\approx 100$ & $\mathrm{~g} \mathrm{~cm}^{2} \mathrm{~s}^{-3} \mathrm{~mA}^{-1}$ & $\approx 10 \mathrm{mV}$ \\
\hline
\end{tabular}

Table A2. Main parameter values in the aorta expressed in $\{c m, g, s, m A\}$ (and recalled in SI units). $\bar{u}_{\text {max }}$ denotes the maximum mean blood velocity at the inlet (during systole).

\begin{tabular}{llll}
\hline & Parameters & Value & units \\
\hline $\boldsymbol{\sigma}_{\mathrm{T}}$ & tissue conductivity & $6.10^{-4}$ & $S \mathrm{~cm}^{-1}$ \\
$\boldsymbol{\sigma}_{\mathrm{bl}}$ & blood conductivity & $6.2510^{-3}$ & $S \mathrm{~cm}^{-1}$ \\
$\boldsymbol{\sigma}_{\text {bone }}$ & bone conductivity & $5.6510^{-5}$ & $S \mathrm{~cm}^{-1}$ \\
$\boldsymbol{\sigma}_{l}$ & lung conductivity & $5.10^{-4}$ & $S \mathrm{~cm}$ \\
$\phi_{\mathrm{T}}$ & electric potential & $\approx 10$ & $\mathrm{mV}$ \\
\hline
\end{tabular}

Table A3. Main parameter values in the body.

simulation of the electromechanical activity of the heart. In Functional Imaging and Modeling of the Heart, number 5528 in Lecture Notes in Computer Science, pages 357-365. Springer-Verlag, 2009.

[5] J. M. Chia, S. E. Fischer, S. A. Wickline, and C. H. Lorenz. Performance of QRS detection for cardiac magnetic resonance imaging with a novel vectorcardiographic triggering method. Journal of Magnetic Resonance Imaging, 12(5):678-688, 2000.

[6] A. Despopoulos and S. Silbernagl. Color atlas of physiology. Number ptie. 471 in Thieme flexibook. Thieme, 2003.

[7] L. Formaggia, A. Quarteroni, and A. Veneziani, editors. Cardiovascular Mathematics. Modeling and simulation of the circulatory system, volume 1 of Modeling, Simulation and Applications. Springer, 2009.

[8] T. Frauenrath, F. Hezel, U. Heinrichs, S. Kozerke, JF. Utting, M. Kob, C. Butenweg, P. Boesiger, and T. Niendorf. Feasibility of cardiac gating free of interference with electro-magnetic fields at 1.5 Tesla, 3.0 Tesla and 7.0 Tesla using an MR-stethoscope. Invest Radiol., 44(9):539-547, 2009.

[9] C.T. Gaffey and T.S. Tenforde. Alterations in the rat electrocardiogram induced by stationary magnetic fields. Bioelectromagnetics, 2:357-370, 1981.

[10] P.L. George, F. Hecht, and E. Saltel. Fully automatic mesh generator for 3d domains of any shape. Impact of Comp. in Sci. ans Eng., 2:187-218, 1990.

[11] J-F. Gerbeau, C. Le Bris, and T. Lelièvre. Mathematical methods for the Magnetohydrodynamics of liquid metals. Numerical Mathematics and Scientific Computation. Oxford University Press, 2006.

[12] R.R. Gold. Magnetohydrodynamic pipe flow. part 1. J. Fluid Mech., 13:505-512, 1962.

[13] A. Gupta, A.R. Weeks, and S.M. Richie. Simulation of elevated T-waves of an ECG inside a static magnetic field (MRI). Biomedical Engineering, IEEE Transactions on, 55(7):1890-1896, 2008.

[14] G. Huiskamp and A van Oosterom. The depolarization sequence of the human heart surface computed from 
measured body surface potentials. IEEE Trans. Biomed. Eng., 35(12):1047-1058, 1988.

[15] W. Kainz, J. Guag, S. Benkler, D. Szczerba, E. Neufeld, V. Krauthamer, J. Myklebust, H. Bassen, I. Chang, N. Chavannes, J.H. Kim, M. Sarntinoranont, and N. Kuster. Development and validation of a magnetohydrodynamic solver for blood flow analysis. Phys. Med. Biol., 55(23):7253, 2010.

[16] Y. Kinouchi, H. Yamaguchi, and T.S. Tenforde. Theoretical analysis of magnetic field interactions with aortic blood flow. Bioelectromagnetics, 17:21-32, 1996.

[17] J. Malmivuo and R. Plonsey. Principles and Applications of Bioelectric and Biomagnetic Fields. Oxford University Press, 1995.

[18] V. Martin, A. Drochon, O. Fokapu, and J-F. Gerbeau. MagnetoHemoDynamics in Aorta and Electrocardiograms. Research Report RR-7747, INRIA, September 2011.

[19] V. Martin, O. Fokapu, A. Drochon, and J-F. Gerbeau. Magnetohemodynamics effect on electrocardiograms. In Functional Imaging and Modeling of the Heart. Springer-Verlag, 2011, to appear.

[20] C.C. Mitchell and D.G. Schaeffer. A two-current model for the dynamics of cardiac membrane. Bulletin Math. Bio., 65:767-793, 2003.

[21] P. Moireau, N. Xiao, M. Astorino, C. Figueroa, D. Chapelle, C. Taylor, and J-F. Gerbeau. External tissue support and fluid-structure simulation in blood flows. Biomechanics and Modeling in Mechanobiology, pages 1-18, 2011. 10.1007/s10237-011-0289-z.

[22] R. Moreau. Magnetohydrodynamics. Kluwer Academic Publishers, 1990.

[23] G.M. Nijm, S. Swiryn, A.C. Larson, and A.V. Sahakian. Characterization of the magnetohydrodynamic effect as a signal from the surface electrocardiogram during cardiac magnetic resonance imaging. In Computers in Cardiology, 2006, pages $269-272$, sept. 2006.

[24] G.M. Nijm, S. Swiryn, A.C. Larson, and A.V. Sahakian. Extraction of the magnetohydrodynamic blood flow potential from the surface electrocardiogram in Magnetic Resonance Imaging. Medical and Biological Engineering and Computing, 46(7):729-733, 2008.

[25] G.M. Nijm, S. Swiryn, A.C. Larson, and A.V. Sahakian. Inhomogeneous human torso model of magnetohydrodynamic blood flow potentials generated in the mr environment. In Computers in Cardiology, 2008, pages $9-12$, sept. 2008.

[26] M. Potse, B. Dubé, J. Richer, A. Vinet, and R. M. Gulrajani. A comparison of monodomain and bidomain reaction-diffusion models for action potential propagation in the human heart. Biomedical Engineering, IEEE Transactions on, 53(12):2425-2435, 2006.

[27] F.B. Sachse. Computational Cardiology: Modeling of Anatomy, Electrophysiology, and Mechanics. SpringerVerlag, 2004.

[28] J. Sundnes, G.T. Lines, X. Cai, B.F. Nielsen, K.-A. Mardal, and A. Tveito. Computing the electrical activity in the heart. Springer-Verlag, 2006.

[29] T.S. Tenforde. Magnetically induced electric fields and currents in the circulatory system. Progress in Biophysics and Molecular Biology, 87:279-288, 2005.

[30] T.S. Tenforde, C.T. Gaffey, B.R. Moyer, and T.F. Budinger. Cardiovascular alterations in Macaca monkeys exposed to stationary magnetic fields: experimental observations and theoretical analysis. Bioelectromagnetics, 4:1-9, 1983.

[31] Z. Tse, Ch. Dumoulin, G. Clifford, M. Jerosch-Herold, D. Kacher, R. Kwong, W. Stevenson, and E. Schmidt. 12-lead ECG in a 1.5 Tesla MRI: Separation of real ECG and MHD voltages with adaptive filtering for gating and non-invasive cardiac output. Journal of Cardiovascular Magnetic Resonance, 12(Suppl 1):O95, 2010. 